\title{
Fabrication and Optical Characterization of Polyvinylidene Fluoride/Neodymium Oxide Nanocomposite Films
}

\author{
Ashok Batra1, Adina Showe1, James Sampson'1, Aschalew Kassu², Michael Curley', Kuzhivelil Arun² \\ ${ }^{1}$ Materials Science Group, Department of Physics, Chemistry, and Mathematics, Alabama A \& M University, Normal (Huntsville), \\ AL, USA \\ ${ }^{2}$ Department of Mechanical, Civil Engineering and Construction Management, Alabama A \& M University, Normal (Huntsville), \\ AL, USA \\ ${ }^{3}$ Department of Physics, Sree Kerala Varma College, Thrissur, Kerala, India \\ Email: ashok.batra@aamu.edu
}

How to cite this paper: Batra, A., Showe, A., Sampson, J., Kassu, A., Curley, M. and Arun, K. (2019) Fabrication and Optical Characterization of Polyvinylidene Fluoride/ Neodymium Oxide Nanocomposite Films. Open Access Library Journal, 6: e5979.

https://doi.org/10.4236/oalib.1105979

Received: December 7, 2019

Accepted: December 24, 2019

Published: December 27, 2019

Copyright () 2019 by author(s) and Open Access Library Inc.

This work is licensed under the Creative Commons Attribution International License (CC BY 4.0).

http://creativecommons.org/licenses/by/4.0/

\begin{abstract}
Polyvinylidene fluoride (PVDF) is employed in a wide range of devices based on its excellent mechanical, optical, high thermal, piezoelectric, pyroelectric, and ferroelectric characteristics. In the current investigation, the pristine and neodymium oxide $\left(\mathrm{Nd}_{2} \mathrm{O}_{3}\right)$ nanoparticles embedded polyvinylidene fluoride (PVDF) thick films were prepared via solution casting method. The $\mathrm{Nd}_{2} \mathrm{O}_{3}$ nanoparticles were synthesized via hydrothermal technique. The functional groups were identified in the nanocomposite films via infrared vibrational spectroscopy. It revealed the presence of ferroelectric $\beta$-phase in the annealed nanocomposite films. All-important optical constants have been determined for the first time via UV-VIS transmission spectroscopy for the nanocomposite films in the ferroelectric phase.
\end{abstract}

\section{Subject Areas}

Atomic Physics, Modern Physics

\section{Keywords}

PVDF, Neodymium Oxide, Nanocomposite Films, Optical Characterization

\section{Introduction}

Ferroelectric materials are called "smart materials" as they can be configured to store, release, or interconvert electrical and mechanical energy in a well-controlled manner via their respective characteristics. They have large piezoelectric com- 
pliances, pyroelectric coefficients, dielectric susceptibilities, and electro-optic properties. Ferroelectric polyvinylidene fluoride (PVDF) is utilized in a wide range of devices due to their excellent mechanical properties, optical, high thermal and chemical stability, piezoelectricity, pyroelectricity, and ferroelectric responses [1]. In the present investigation, the pristine and neodymium oxide $\left(\mathrm{Nd}_{2} \mathrm{O}_{3}\right)$ nanoparticles embedded polyvinylidene fluoride (PVDF) thick films were fabricated via solution casting/intercalation technique. The $\mathrm{Nd}_{2} \mathrm{O}_{3}$ nanoparticles were synthesized via hydrothermal technique. The Fourier Transform Infrared Spectroscopy (FTIR) has been recorded in the range 400 to $4000 \mathrm{~cm}^{-1}$, and functional groups were identified in the nanocomposite films. Infrared vibrational spectroscopy (FTIR) revealed the presence of ferroelectric $\beta$-phase intrinsically in the annealed nanocomposite films. All-important optical constants have been determined for the first time via UV-VIS transmission spectroscopy for the nanocomposite films in the ferroelectric phase.

With the rapid growth of technologies, electroactive polymers (EAPs) are gaining an immense amount of interest as smart materials. There are different EAPs, namely, Nylon-11, polylactide and aniline pentamer copolymer, poly(lactic-co-glycolic acid) (PLGA), and poly(vinylidene fluoride) (PVDF) and its copolymers with trifluoroethylene (TrFE) [2]-[7]. Amongst them, PVDF and PDVF-TrFE exhibit the best electroactive properties, such as piezo-, pyro- and ferroelectricity and optoelectronic. As a result, PVDF and its copolymers are the polymer of choice for the increasing number of possible microelectronics applications, such as electro-optic transducers, waveguides, sensors, actuators, energy harvesting, electro-optic memory, and biomimetic robotic, etc. [2]. PVDF is inherently polar. With respect to the carbon atom in the unit, the hydrogen atoms are positively charged, and the fluorine atoms are negatively charged. The repeat unit of $\left(-\mathrm{CH}_{2}-\mathrm{CF}_{2}\right)$ or $\left.\mathrm{CH}_{2} \mathrm{CF}_{2}\right)$ n, in which the carbon-hydrogen bonds with the electrically polar carbon-fluorine, can take up a number of stable configurations determined by the treatment of the polymer. PVDF can be crystallized into at least four different polymorphous phases, depending on the film preparation conditions. Two forms $\beta$ (or I) and $\alpha$ (or II) are of particular importance. In form $\alpha$, PVDF (formed from slow-cooled melts or an acetone solution) is non-piezoelectric, the bonds are in a trans-gauche-trans-gauche configuration, and the molecules are stacked, giving a nonpolar unit cell. Form $\alpha$ can be converted into form $\beta$ by stretching and electrically poling with a suitable field [3]. In form $\beta$, the molecular groups are in an all-trans configuration, and the molecules are assembled to give a polar unit cell. Form $\beta$ is orthorhombic with point group A $\mathrm{mm}^{2}$.

In the recent past, PVDF has attracted interest in the photonics area because of the possibility to be used as hosts for lanthanides photonics ions [4]. In designing the above mentioned emerging optical devices and sensors, the variation of all the standard basic parameters and constants with photon energy are warranted and justified to be investigated. However, few works can be found in the 
literature about the study of the complete optical properties of $\mathrm{Nd}_{2} \mathrm{O}_{3}$ nanoparticles embedded PVDF thick films in UV-Vis range [5]. Thus, this publication presents all the essential optical constants, including optical bandgap of nanocomposite PVDF films with various amounts of $\mathrm{Nd}_{2} \mathrm{O}_{3}$ nanoparticles.

\section{Experimental}

\subsection{Preparation of $\mathrm{Nd}_{2} \mathrm{O}_{3}$ Nanoparticles}

$\mathrm{Nd}_{2} \mathrm{O}_{3}$ nanoparticles were prepared using 0.1 molar aqueous solution of neodymium chloride hexahydrate and 0.5 molar aqueous solution of sodium hydroxide without any surfactants. An equal amount of the precursors were mixed and cooked in the Teflon lined autoclave at $200^{\circ} \mathrm{C}$ for 9 hours and brought into ambient temperature by slow cooling. The precipitate neodymium hydroxide is centrifuged, cleaned thoroughly with Millipore water several times and heated to $300^{\circ} \mathrm{C}$ in the air in a muffle furnace resulting in the formation of neodymium oxide leaving behind water vapors according to the reaction.

$$
\begin{gathered}
\mathrm{NdCl}_{3} \cdot 6 \mathrm{H}_{2} \mathrm{O}+3 \mathrm{NaOH} \rightarrow \mathrm{Nd}(\mathrm{OH})_{3}+3 \mathrm{NaCl}+6 \mathrm{H}_{2} \mathrm{O} \\
2 \mathrm{Nd}(\mathrm{OH})_{3} \rightarrow \mathrm{Nd}_{2} \mathrm{O}_{3}+3 \mathrm{H}_{2} \mathrm{O} \uparrow
\end{gathered}
$$

\subsection{Preparation of PVDF-NdO Films}

$500 \mathrm{mg}$ of PVDF is dissolved in $20 \mathrm{ml}$ of DMF solvent and $\mathrm{Nd}_{2} \mathrm{O}_{3}$ nanoparticles in the different $\mathrm{wt} \%$ ratio ( $2 \mathrm{wt} \%$ and $4 \mathrm{wt} \%$; coded as $\mathrm{Nd} 2$ and $\mathrm{Nd} 4$ ) is added to this solution with vigorous agitation to obtain a clear solution of the mixture. It is then poured in the petri dish and kept at $60^{\circ} \mathrm{C}$ in a vacuum oven for the evaporation of the solvent. Self-standing films of the PVDF- $\mathrm{Nd}_{2} \mathrm{O}_{3}$ composite with different $w t \%$ without any inclusions were used for different studies.

\subsection{Optical Studies on PVDF-NdO Films}

FTIR spectra of the films have been recorded in the range 400 to $4000 \mathrm{~cm}^{-1}$. The optical transmittance in the UV-VIS region of the samples was recorded using Hitachi U-2900 (2J1-002) dual-beam spectrophotometer in the wavelength range $200-800 \mathrm{~nm}$. The surrounding medium was air and all the measurements were performed at room temperature.

The measured transmittance $(T)$ was used to calculate the absorption coefficient $(\alpha)$ using the formula:

$$
\alpha=\frac{2.303 \log \left(\frac{1}{T}\right)}{t}
$$

where, $t$ is the thickness of the sample. It is known that, if multiple reflections are neglected, the reflectance $R$ of the sample can be calculated from the measured values of the transmittance $T$ and absorbance $A$ using the following equation: 


$$
R=1-(T+A)
$$

The refractive index $(n)$ of the material was calculated from reflectance values using the expression:

$$
n=\frac{1+R^{2}}{1-R^{2}}
$$

The optical conductivity is one of the powerful tools for studying the electronic states in the material and is given by the following relation:

$$
\text { Optical Conductivity }=\frac{\alpha n c}{4 \pi}
$$

where, $c$ is the speed of light.

The optical density is given by:

$$
\text { Optical Density }=\alpha * t
$$

The extinction coefficient $k$ can be obtained from the equation:

$$
k=\frac{\alpha \lambda}{4 \pi}
$$

\section{Results and Discussions}

FTIR spectra of the film are illustrated in Figure 1. The FTIR analysis revealed the electroactive $\beta$ phase of the fabricated films at around $845 \mathrm{~cm}^{-1}$.

Figure 2(a) to Figure 2(b) show the absorption and transmittance spectra of $\mathrm{NdO}$ films, respectively. The figure indicates that the transmittance of PVDF films decreases with the addition of $\mathrm{Nd}_{2} \mathrm{O}_{3}$ nanoparticles. The decrease in transmittance may be due to light scattering of caused by nanoparticles. Figure 3 shows the variation of absorption coefficient with wavelength of $\mathrm{Nd}-0, \mathrm{Nd}-2$ and Nd-4 films fabricated.

The complex refractive index is the representation of the optical constants of

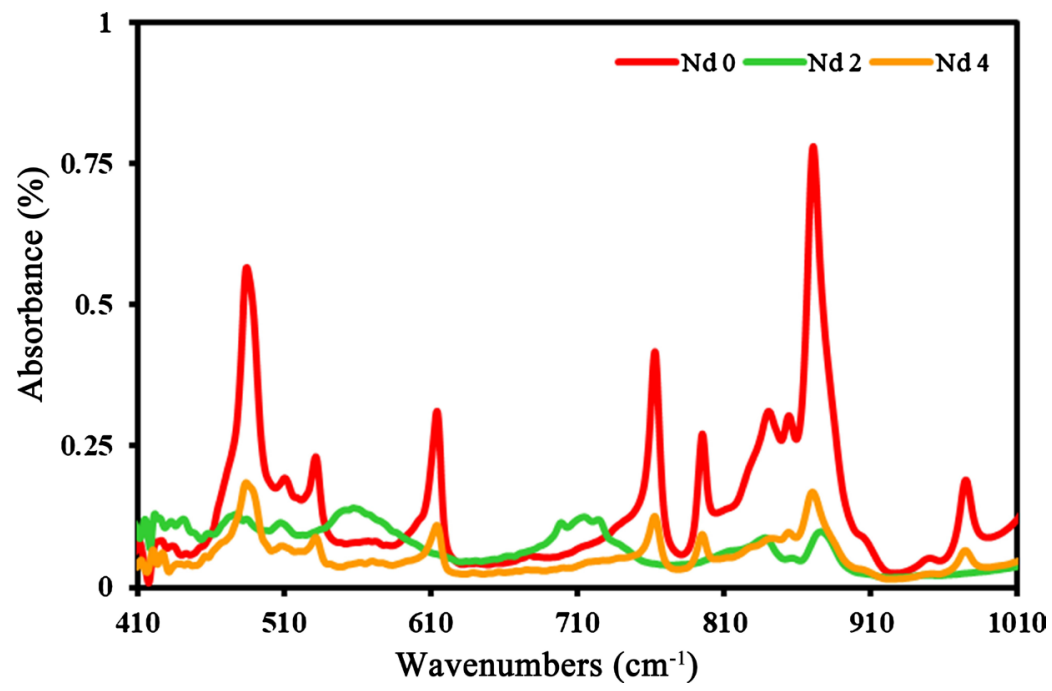

Figure 1. FTIR spectra of Nd-0, Nd-2 and Nd-4 samples. 

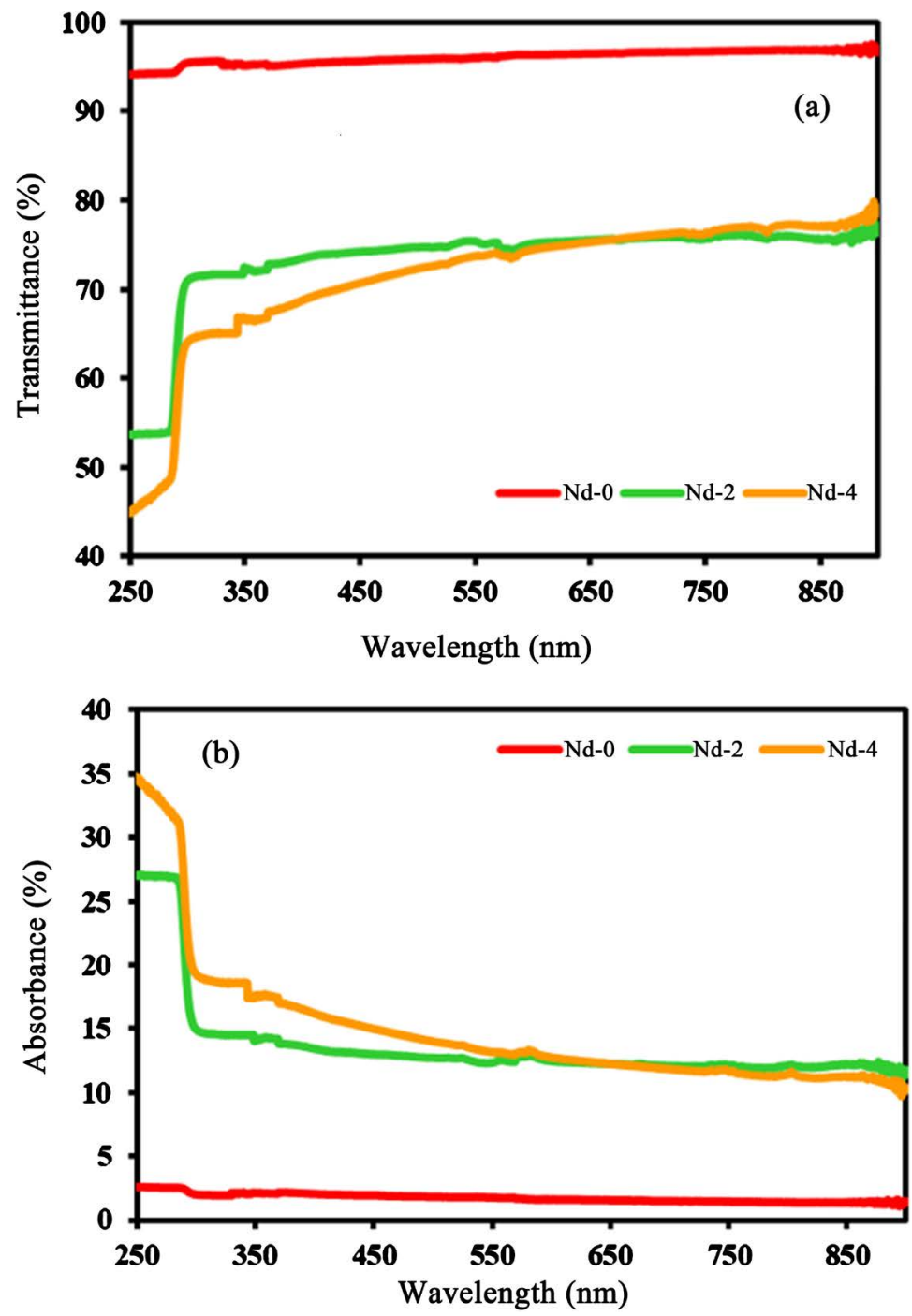

Figure 2. Variation of transmittance (T) (a), and absorbance (b) of the thin films.

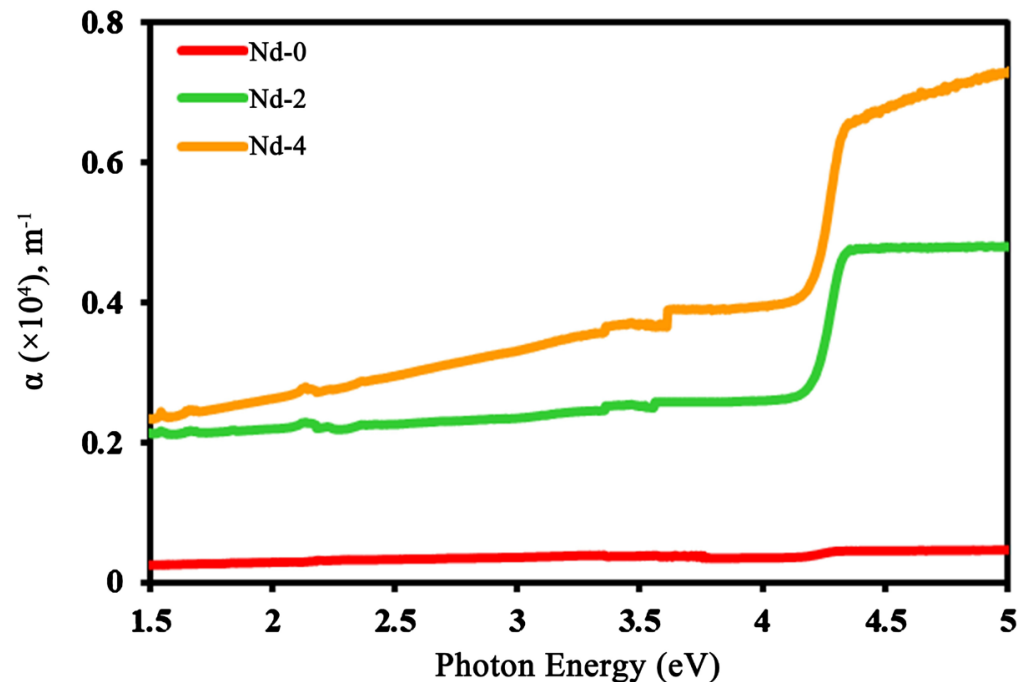

Figure 3. Variation of absorption coefficient $(\alpha)$ with the photon energy of pristine PVDF thin film. 
material and in represented by $n^{*}=n+i k$. The real part " $n$ " is the index of refraction, defines the phase velocity of light in material: $v=c / n$. where $v$ is the speed of light in materials and $c$ is the speed of light in vacuum. The imaginary part " $k$ " is the extinction coefficient, determines how fast the amplitude of the wave decreases. The variation of $n$ and $k$ both decrease with wavelength as shown in Figure 4(a) and Figure 4(b), respectively. This may be due to a change in density and/or the polarization in the film.

The optical conductivity is one of the powerful tools for studying the electronic states in materials. The plot between the optical conductivity (O.C) and optical density (OD) against photon energy is depicted in Figure 5(a) and Figure 5(b), respectively. The spectrum indicates that optical conductivity increases with the increase of photon energy. The behavior of optical density (O.D) is similar to the variation of O.C with photon energy in pure PVDF film.

The dielectric function $\left(\varepsilon T=\varepsilon^{\prime}+i \varepsilon^{\prime \prime}\right)$ is a complex quantity and a fundamental intrinsic property of the material which consists of both the real and imaginary parts $\left(\varepsilon^{\prime}\right.$ and $\left.\varepsilon^{\prime \prime}\right)$. The real part indicates how the speed of light in the material can be slowed down while the imaginary part deals with the absorption of energy by a dielectric from the electric field due to dipole motion.
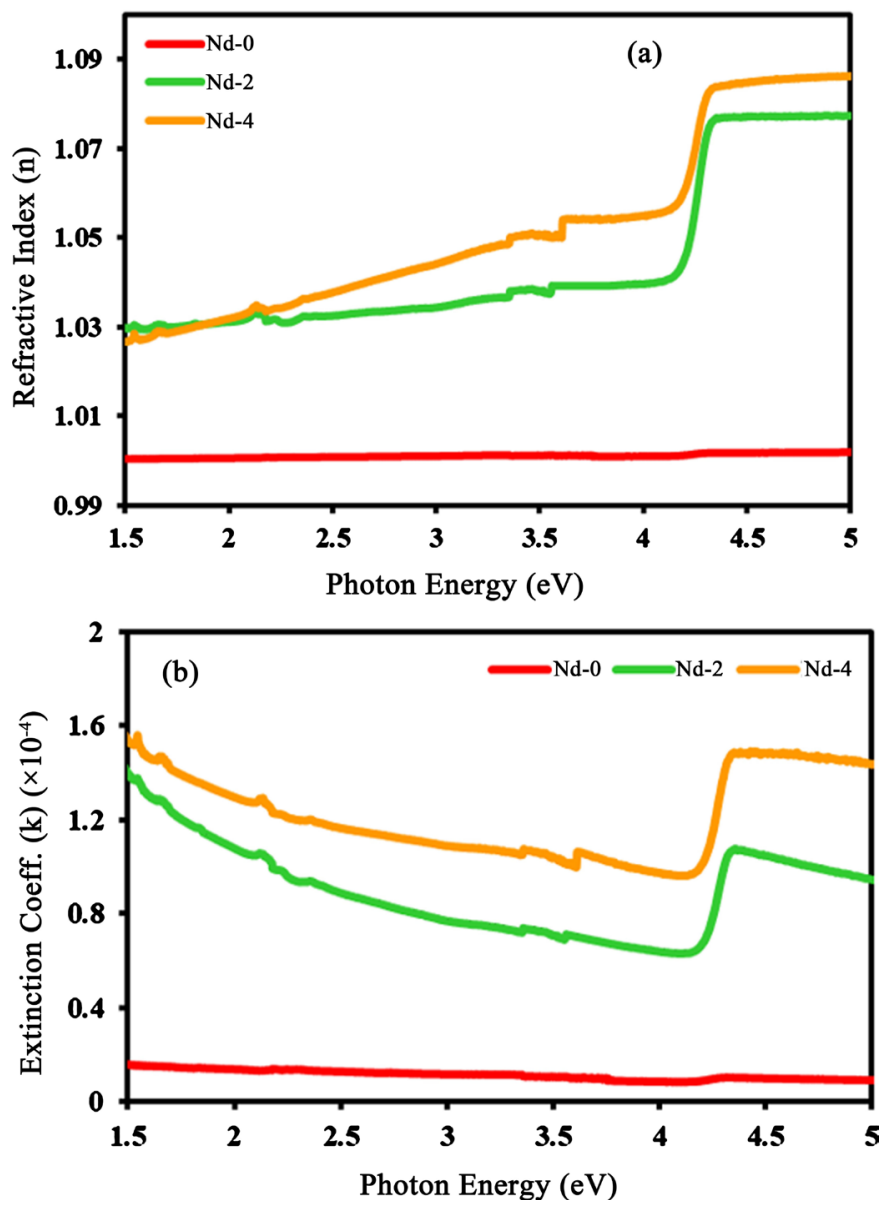

Figure 4. Variation of refractive index ( $n)(\mathrm{a})$, and extinction coefficient $(k)(\mathrm{b})$ as a function of the photon energy of PVDF poled thin film. 

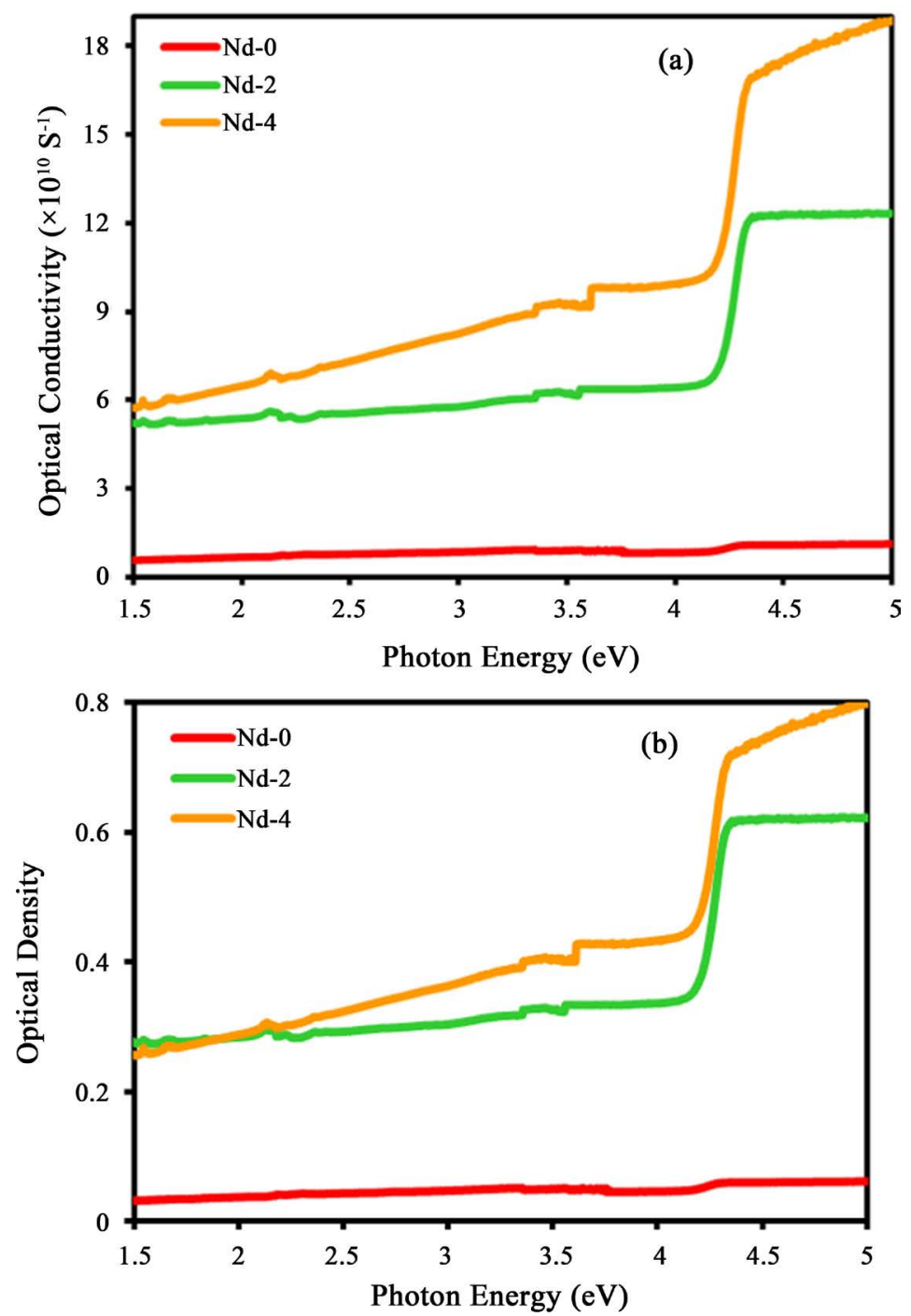

Figure 5. Variation of optical conductivity (O.C) (a) and optical density (O.D) (b) as a function of the photon energy of pure PVDF thin film.

From the optical dielectric curve shown in Figure 6, it is evident that $\varepsilon^{\prime}$ increases with an increase of photon energy. The calculated dielectric loss tangent $(\tan \delta)$ of the PVDF film represents the depletion of the electrical energy [2] [6]. The study of the absorption edge is essential in connection with the theory of electronic structure, which leads to the prediction of whether the band structure is affected near the band extreme. So the main aim is to determine the magnitude and the nature of the optical energy band gap of pure PVDF thin film. The optical energy gap of the films is determined by the absorption spectra. According to the Tauc relation, the absorption coefficient $\alpha$ for the material is given by [2] [6]:

$$
A h v=A(h v-E g)^{n}
$$

where $E g$ is the optical energy gap; $A$ is a constant and is different for different transitions; $h v$, is the energy of photon; and $n$ is an index which assumes the 

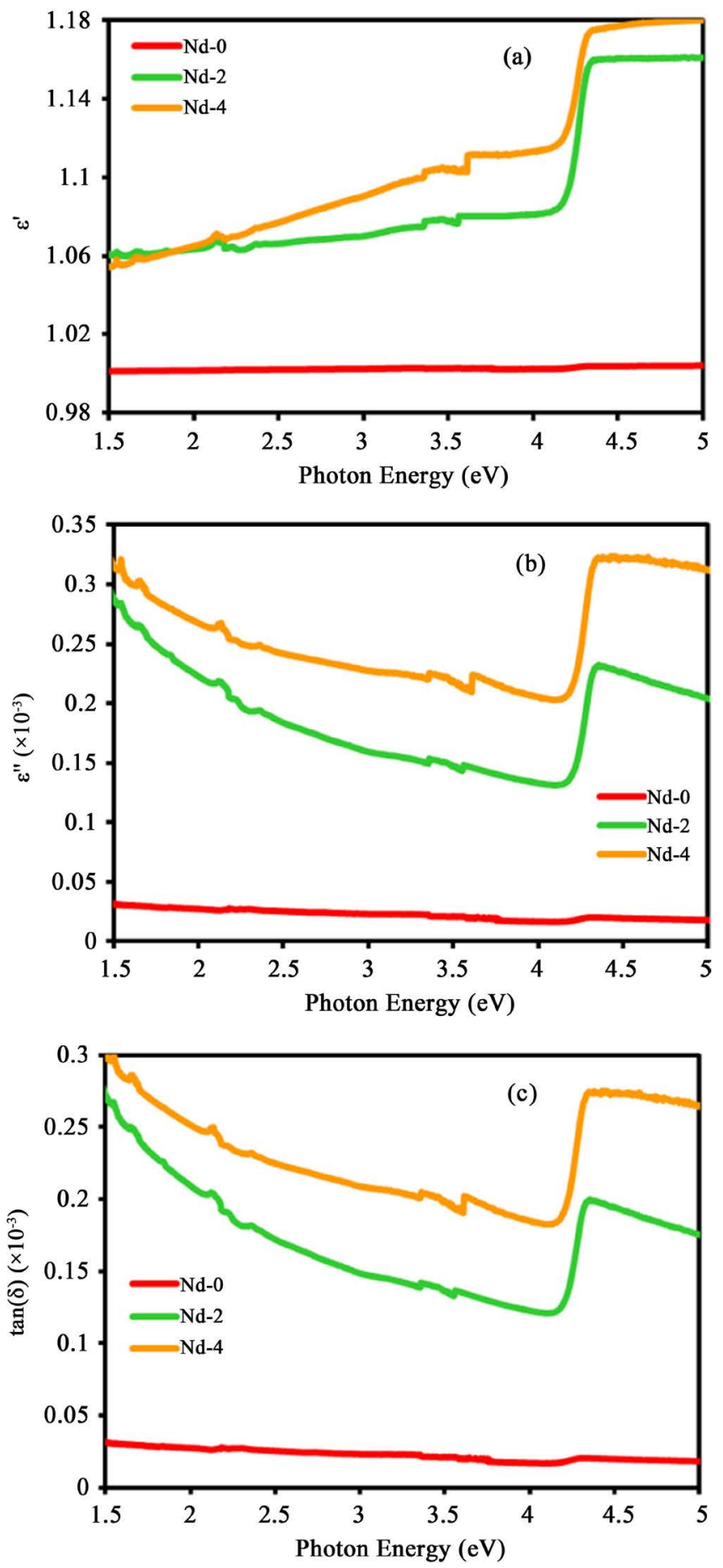

Figure 6. Variation of $\varepsilon^{\prime}(\mathrm{a}), \mathcal{\varepsilon}^{\prime \prime}(\mathrm{b})$, and $\tan \delta(\mathrm{c})$ as a function of photon energy.

values $1 / 2,3 / 2,2$ and 3 depending on the nature of electronic transitions responsible for absorption. Thus, the bandgap is obtained by plotting $(\alpha h v)^{1 / n}$ versus $h v$ in the high absorption range followed by extrapolating the linear region of the plots to $(\alpha h v)^{1 / n}=0$. The analyses of our data showed that the plots of $(\alpha h v)^{1 / n}$ against $h v$ with $n=1 / 2$. This indicates the allowed direct transition is responsible for the inter-band transition in the doped crystal. Figures 7(a)-(c) show the 

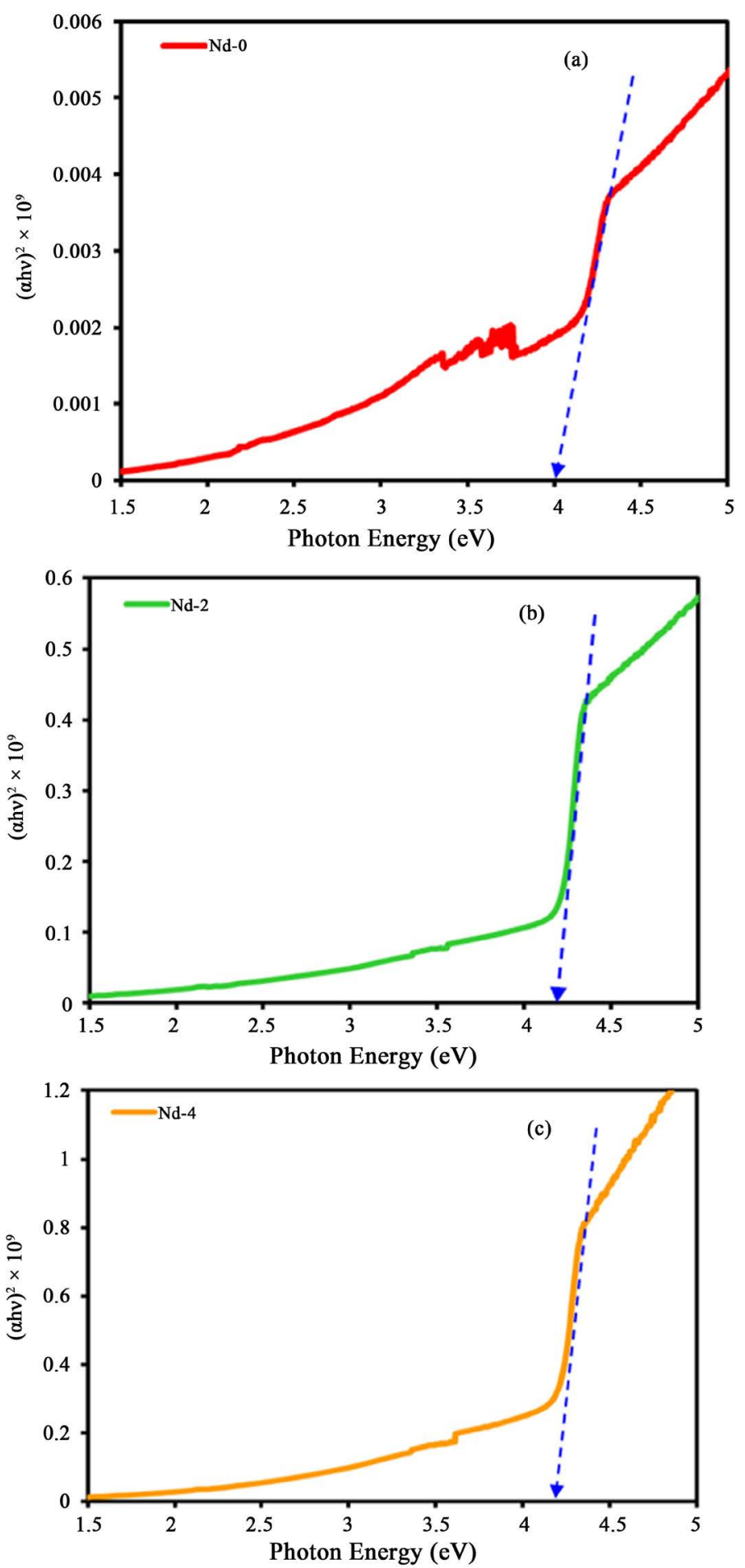

Figure 7. Direct and indirect optical band gap as a function of photon energy for samples Nd-0 (a), Nd-2 (b), and Nd-4 (c).

plot of $(\alpha h v)^{2}$, against photon energy $h v$ for the Nd-0, Nd-2 and Nd-4 films. As shown in Figure 7, the values of the optical bandgaps $(E g)$ for $(\alpha h v)^{2}$, of pure PVDF film (Nd-0), Nd-2 and Nd-4, were found to be 4.0, 4.2, and $4.3 \mathrm{eV}$ respectively. Table 1 summarizes the optical constants obtained at the wavelengths 500 
Table 1. Summary of optical constants for samples Nd-0, Nd-2, and Nd-4 at 500, 600 and $700 \mathrm{~nm}$.

\begin{tabular}{|c|c|c|c|c|c|c|c|}
\hline \multicolumn{8}{|c|}{ Optical Constants } \\
\hline \multirow{2}{*}{ Sample } & $n$ & $K\left(\times 10^{-4}\right)$ & O.D. & O.C. $(\times 1010)$ & $\varepsilon^{\prime}$ & $\varepsilon^{\prime \prime}\left(\times 10^{-3}\right)$ & $\tan (\delta)\left(\times 10^{-3}\right)$ \\
\hline & \multicolumn{7}{|c|}{ At $\lambda=500 \mathrm{~nm}(2.48 \mathrm{eV})$} \\
\hline Nd-0 & 1.001 & 0.128 & 0.042 & 0.766 & 1.002 & 0.026 & 0.025 \\
\hline $\mathrm{Nd}-2$ & 1.032 & 0.895 & 0.292 & 5.542 & 1.066 & 0.185 & 0.173 \\
\hline \multirow[t]{2}{*}{ Nd-4 } & 1.038 & 1.169 & 0.323 & 7.274 & 1.076 & 0.243 & 0.225 \\
\hline & \multicolumn{7}{|c|}{ At $\lambda=600 \mathrm{~nm}(2.066 \mathrm{eV})$} \\
\hline $\mathrm{Nd}-0$ & 1.001 & 0.134 & 0.036 & 0.668 & 1.002 & 0.270 & 0.027 \\
\hline $\mathrm{Nd}-2$ & 1.032 & 1.053 & 0.286 & 5.425 & 1.064 & 0.217 & 0.204 \\
\hline \multirow[t]{2}{*}{$\mathrm{Nd}-4$} & 1.033 & 1.276 & 0.294 & 6.584 & 1.067 & 0.264 & 0.247 \\
\hline & \multicolumn{7}{|c|}{ At $\lambda=700 \mathrm{~nm}(1.771 \mathrm{eV})$} \\
\hline $\mathrm{Nd}-0$ & 1.001 & 0.143 & 0.033 & 0.614 & 1.001 & 0.290 & 0.029 \\
\hline $\mathrm{Nd}-2$ & 1.030 & 1.192 & 0.278 & 5.256 & 1.061 & 0.246 & 0.231 \\
\hline Nd-4 & 1.029 & 1.384 & 0.273 & 6.097 & 1.060 & 0.285 & 0.269 \\
\hline
\end{tabular}

$\mathrm{nm}, 600 \mathrm{~nm}$ and $700 \mathrm{~nm}$ respectively.

\section{Conclusion}

In this study, optical quality PVDF nanocomposites film with has been fabricated and the optical constants, such as the direct and indirect optical bandgaps $(E g)$, absorption coefficient $(\alpha)$, refractive index $(n)$, extinction coefficient $(k)$, and real and imaginary dielectric constants $\left(\varepsilon^{\prime}\right.$ and $\left.\varepsilon^{\prime \prime}\right)$ and the electric loss tangent $(\tan \delta)$ were calculated to analyze the optical properties of PVDF thick films with embedded $\mathrm{Nd}_{2} \mathrm{O}_{3}$ nanoparticles. The results of the UV-VIS spectroscopy measurements show the absorption and transmission property of the PVDF films, and the Raman analysis revealed the electroactive $\beta$ phase of the fabricated films at around $845 \mathrm{~cm}^{-1}$.

\section{Acknowledgements}

The authors gratefully acknowledge support for this work through the title III program at Alabama A \& M University. Authors thank Dr. M. D. Aggarwal for his support and encouragement.

\section{Conflicts of Interest}

The authors declare no conflicts of interest regarding the publication of this paper.

\section{References}

[1] Kepler, R.G. (1995) Ferroelectric Polymers: Chemistry, Physics, and Applications. 
Ferroelectric, Pyroelectric, and Piezoelectric Properties of Poly(Vinylidene Fluoride). Nalwa, H.S., Ed. Marcel Dekker, New York, 183-232.

[2] Ruan, L., Yao, X., Chang, Y., Zhou, L., G. Qin, G. and Zhang, X. (2018) Properties and Applications of the $\beta$-Phase Poly(Vinylidene fluoride). Polymers, 10, 228.

https://doi.org/10.3390/polym10030228

[3] Shanshool, H.M., Yahaya, M., Yunus, W. and Abdullah, I.Y. (2015) Optical Properties of PVDF/ZnO Nanocomposites. International Journal of Technical Research and Applications, 23, 51-58.

[4] Aguiar, L., Silva, C., Anderson, C., Rinaldi, A., Botero, E., Carvalho, C., Guo, R., Falcao, E. and Bhalla, A. (2017) Improving the Optical Properties of Poly(Vinylidene Fluoride) (PVDF) Films by Doping with $\mathrm{Nd}^{3+}$ Compound: Synthesis and Characterization. Proceedings of IMF-2017, San Antanio, TX, 4-8 September 2017.

[5] Ismail, A.M., Mohammed, M.I. and Fouad, S.S. (2018) Optical and Structural Properties of Polyvinylidene Fluoride (PVDF)/Reduced Graphene Oxide (RGO) Nanocomposites. Journal of Molecular Structure, 1170, 51-59.

https://doi.org/10.1016/j.molstruc.2018.05.083

[6] Sebastian, M.T. (2008) Dielectric Materials for Wireless Communication. Elsevier Ltd., UK.

[7] Indolia, A. and Gaur, M. (2013) Optical Properties of Solution Grown PVDF-ZnO Nanocomposite Thin Films. Journal of Polymer Research, 20, 43-50.

https://doi.org/10.1007/s10965-012-0043-y 